\title{
Bifidobacterium Iongum and Lactobacillus helveticus Synergistically Suppress Stress-related Visceral Hypersensitivity Through Hypothalamic- Pituitary-Adrenal Axis Modulation
}

\author{
Afifa Ait-Belgnaoui, ${ }^{1,2 *}$ Isabelle Payard, ${ }^{1}$ Claire Rolland, ${ }^{2}$ Cherryl Harkat, ${ }^{1}$ Viorica Braniste, ${ }^{3}$ Vassillia Théodorou, ${ }^{1}$ and \\ Thomas A Tompkins ${ }^{2}$ \\ ${ }^{1}$ Neuro-Gastroenterology and Nutrition team, TOXALIM, UMR 1331-INRA/INP/UPS, Toulouse, France; ${ }^{2}$ Lallemand Health Solutions, Montreal, \\ Canada; and ${ }^{3}$ Department of Microbiology, Tumor and Cell Biology, Karolinska Institute, Stockholm, Sweden
}

\section{Background/Aims}

Visceral pain and hypothalamic-pituitary-adrenal axis (HPA) dysregulation is a common characteristic in irritable bowel syndrome (IBS) patients. Previously, we reported that a probiotic formulation (Lactobacillus helveticus R0052 and Bifidobacterium longum R0175) prevents chronic stress-mediated brain function abnormalities by attenuating the HPA axis response. Here, we compared the effect between different probiotic treatments on the perception of visceral pain during colorectal distension (CRD) following a chronic stress and the consequences to the activity of the HPA axis.

\section{Methods}

After a 2-week treatment with a combined probiotic formulation, or L. helveticus or B. longum alone in stressed mice, the visceral pain in response to CRD was recorded. The expression of glucocorticoid receptors was determined in the different brain areas involved in the stress response (hypothalamus, hippocampus, and prefrontal cortex). The plasma levels of stress hormones were also measured.

\section{Results}

A pretreatment using the combination of probiotic formulation significantly reduces the chronic stress-induced visceral hypersensitivity respectively at $0.06,0.08$, and $0.10 \mathrm{~mL} C R D$ volume. However, a single probiotic (B. longum or L. helveticus) administration is less effective in reducing visceral pain in stressed mice. Moreover, the expression of the glucocorticoid receptor mRNA was consistently up-regulated in several brain areas after pretreatment with a combined probiotic, which correlated with the normalization of stress response compared to the inconsistent effects of a single probiotic.

\section{Conclusion}

The combination of $L$. helveticus and $B$. longum is more effective in regulating glucocorticoid negative feedback on the HPA axis than probiotic alone and subsequently in treating stress-induced visceral pain.

(J Neurogastroenterol Motil 2018;24:138-146)

Key Words

Chronic stress; Glucocorticoid receptors; Probiotics; Visceral pain

Received: October 13, 2016 Revised: June 13, 2017 Accepted: July 12, 2017

(.) This is an Open Access article distributed under the terms of the Creative Commons Attribution Non-Commercial License (http://creativecommons. org/licenses/by-nc/4.0) which permits unrestricted non-commercial use, distribution, and reproduction in any medium, provided the original work is properly cited.

*Correspondence: Afifa Ait-Belgnaoui, PhD

19 rue des Briquetiers, B.P 59, 31702 Blagnac cedex, France

Tel: +33-0-615572179, Fax: +33-0-562745500, E-mail: afifa.ait.belgnaoui@gmail.com 


\section{Introduction}

Chronic or recurrent abdominal pain, bloating, and alteration of stool frequency and consistency, are frequent symptoms associated with the irritable bowel syndrome (IBS). In Western countries, $25 \%$ of medical consultations in gastroenterology are due to visceral hypersensitivity. ${ }^{1}$ Although the IBS does not represent a lifethreatening condition, it impacts the patient quality of life, and the management of this disorder represents an important economic burden. ${ }^{2}$ The pathogenesis of visceral pain in association with clinical symptoms of IBS may occur within the peripheral and the central nervous systems. Evidence suggests that physical or psychological stress can aggravate IBS symptoms ${ }^{3}$ and have a profound effect on visceral pain ${ }^{4}$ via affecting the brain-gut interaction, central pain perception, abnormalities of hypothalamic-pituitary-adrenal (HPA) axis, and other brain structures. ${ }^{5,6}$ In HPA axis activity, a central stress response is regulated by circulating glucocorticoid feedback ${ }^{7}$ via binding to mineralocorticoid and glucocorticoid receptors (GR) at multiple sites in the brain including the hypothalamus, anterior pituitary, hippocampus, and prefrontal cortex. ${ }^{8}$ It is well known that chronic stress attenuates the glucocorticoid negative feedback, disrupts GR expression in the brain-related areas, ${ }^{9}$ and causes chronic abdominal pain. ${ }^{10}$ At the intestinal level, exposure to chronic stress results in maladaptive changes at cellular, structural, functional, and microbial levels, the latter resulting in microbial dysbacteriosis. ${ }^{11}$ Current research reports an altered composition of the intestinal microbiota in IBS patients, ${ }^{12,13}$ and the lower microbial diversity in the intestine is correlated with the severity of IBS symptoms. ${ }^{14}$ Moreover, an animal study has shown that the transplantation of the fecal microbiota from IBS patients into germ-free mice exhibits an increase of the abdominal contractions. ${ }^{15}$ Thus, targeting intestinal microbiota to regulate the visceral pain seems to be an interesting therapeutic approach. Indeed, fecal transplants might be a highly effective treatment of chronic diseases ${ }^{16,17}$ and reduce symptoms in IBS patients. ${ }^{18}$ Dietary intervention by probiotics can also be used in the manipulation of intestinal microbiota. A growing body of clinical trials suggests that probiotic treatment may improve IBS symptoms ${ }^{19,20}$ but their role in the criterion of visceral pain remains unknown. Interestingly, in IBS-like animal models, probiotics such as bifidobacterium or lactobacillus species are able to reduce visceral pain through the regulation of the neural function ${ }^{21-23}$ and normalize the HPA function. ${ }^{24}$

In a previous study we have shown that the probiotic formulation (Lactobacillus helveticus and Bifidobacterium longum) at- tenuates the hyper-responsiveness of the HPA axis and facilitates neurogenesis in the hippocampus. These data suggest that the potential central regulation of stress response by the combination of the 2 probiotics may be due to the regulation of glucocorticoid negative feedback of the HPA axis. ${ }^{25}$ In the current study, we aim to determine whether the attenuated effect of the stress response is due to a specific probiotic strain or to their combined actions. For this purpose, we evaluated the neuroendocrine response to stress and GR expression in specific brain areas. In addition, we evaluated the response of visceral pain to the nociceptive stimuli in mice treated with the probiotic strains, B. longum R0175 or L. helveticus R0052 alone or in combination.

\section{Materials and Methods}

\section{Animals}

C57BL/6J male mice (6-8 weeks old) (Janvier SA, Le Genest St Isle, France) weighing 21-23 g were used. Mice were housed under laboratory conditions $\left(25 \pm 2{ }^{\circ} \mathrm{C}, 12\right.$-hour light-dark cycle), with free access to standard diet and water. All experiments were approved by the local ethical board, Toulouse, France, following the European Union legislation (Council Directive 2010/63/EU).

\section{Preparation of Probiotic}

A combination of probiotic (L. helveticus R0052 and B. longum R0175), and individual probiotic strain L. helveticus R0052 or B. longum R0175 were provided by Lallemand Solutions Santé (Blagnac, France). Before administration, freeze-dried pure bacteria were rehydrated in $0.9 \%$ sodium chloride $(\mathrm{NaCl})$ at a concentration of $10^{9}$ colony-forming units (CFU) per milliliter. The bacterial solution was administered by oral gavage daily for 2 weeks before and during the experimental procedure.

\section{Chronic Stress Protocol}

Repeated exposure to water avoidance stress (WAS) was performed as described previously. ${ }^{25}$ Briefly, the mice were placed individually during a 1-hour period on a plastic platform positioned in the middle of a basin filled with room temperature water. The procedure was repeated for 4 consecutive days. A control group was not exposed to the daily WAS but was placed into a basin without water. The WAS session has been shown to act a psychological stressor causing an increase of stress hormone, ${ }^{26}$ visceral pain, ${ }^{27}$ and intestinal dysbiosis. ${ }^{28}$ 


\section{Plasma Stress Hormone Analysis}

At the end of the chronic stress, blood samples were collected via the facial vein into heparinised tubes and the mice were sacrificed by cervical dislocation. Plasma was obtained after centrifugation and stored at $-80^{\circ} \mathrm{C}$ until further analysis. Enzyme-linked immunosorbent assays (ELISA) were performed to quantify plasma corticosterone and adrenaline/noradrenaline levels (Immunodiagnostic system, Paris, France and LDN, Nordhorn, Germany, respectively).

\section{Gene Expression Studies}

Total RNA from the hypothalamus, hippocampus, and prefrontal cortex was extracted using the Qiazol reagent (Qiagen, Courtaboeuf, France). RNA integrity and quantification were confirmed using a NanoDrop ND2000 (Thermo Scientific, Illkirch, France). Total RNA samples $(2 \mu \mathrm{g})$ were reverse-transcribed using high-capacity cDNA reverse transcription kit (Applied Biosystems, Courtaboeuf, France). Amplicons were performed with IQ SYBR green Supermix (Biorad, Marnes-la-Coquette, France) on the CFX 96 (Biorad). Quantitative polymerase chain reaction (qPCR) data was normalized by TATA-box binding protein (TBP) expression levels and analysed using $2^{-\mathrm{ACT}}$. The following primer sequences were: GR forward 5'-TCTCCTCAGTTCCTAAGGAAGGTC-3' and reverse 5'-CTGACTGGAGTTTCCTTCCCTTT-3'; TBP forward 5'-GGGAGAATCATGGACCAGAA-3' and reverse 5'-GATGGGAATTCCAGGAGTCA-3'.

\section{Electromyography Recording}

Animals were placed under anesthesia (xylasine-ketamine, 5 volume/volume) and electrodes were implanted in the abdominal muscle after an abdominal incision. Seven days later, the intensity of abdominal contractions was determined by recording the abdominal electromyography to graded colorectal distension (CRD), as a measure of visceral hypersensitivity.

Following an adaptation period of 1 hour in the small tunnel, a balloon catheter (Fogarty catheter for arterial embolectomy; Edwards Lifesciences, Nijmegen, The Netherlands) was inserted $2.5 \mathrm{~cm}$ into the colon and CRD was performed as previously described. $^{29}$

\section{Experimental Protocol}

In the first set of experiments, 8 groups of mice ( $\mathrm{n}=8$ mice per group) received orally, for 2 weeks, either the probiotic combination of the 2 bacterial strains (B. longum $+L$. helveticus) or single probiotic (B. longum or $L$. helveticus) at the concentration of
$10^{9} \mathrm{CFU} /$ day. The control groups received $\mathrm{NaCl} 0.9 \%$ (vehicle). All the animals were submitted to WAS or sham stress during 1 hour per day for 4 days. During the WAS or sham stress session, the probiotic or vehicle treatments were maintained. At the end of the stress session, visceral sensitivity was recorded.

In the second set of experiments, the mice were divided into 8 groups ( $\mathrm{n}=8$ mice per group) and received orally either the probiotic combination of 2 bacteria (B. longum + L. helveticus) or a single bacterium (B. longum or L. helveticus) ( $10^{9} \mathrm{CFU} /$ day) or vehicle $(\mathrm{NaCl} 0.9 \%)$ for 2 weeks. Following WAS or sham stress session, blood was collected through the facial vein puncture for measurement of the corticosterone, adrenaline, and noradrenaline. Immediately after blood drawing, the animals were decapitated and the brains removed from the skulls and cooled on ice. The hypothalamus, hippocampus, and prefrontal cortex were quickly dissected, frozen in liquid nitrogen and stored in $-80^{\circ} \mathrm{C}$ until further qPCR analysis.

\section{Statistical Methods}

All data, previously checked for normal distribution, are shown as means \pm SEM. Analysis were evaluated using GraphPad Prism 4 software (GraphPad, San Diego, CA, USA). Abdominal contractions for a 5-minute period during $\mathrm{CRD}$ and plasma hormone concentration were analysed by ANOVA and Tukey tests for post hoc comparisons. Quantitative PCR data was analyzed using Student $t$ test. A $P<0.05$ was considered significant.

\section{Results}

\section{Effect of the Combination of the Probiotics Bifidobacterium longum and Lactobacillus helveticus or Probiotic Alone on Stress-induced Visceral Pain Perception}

In mice, the chronic stress (WAS, $P<0.05$ ) increased significantly the abdominal pain in response to CRD compared with the control group (without chronic stress). The treatment with probiotic combination (B. longum and L. helveticus) significantly reversed chronic stress-induced abdominal pain in response to $\mathrm{CRD}$ at the volume of $0.06 \mathrm{~mL}(48.1 \pm 9.1$ vs $87.5 \pm 14.3, P<0.05), 0.08$ $\mathrm{mL}(49.7 \pm 15.7$ vs $118.6 \pm 12.3, P<0.05)$, and $0.10 \mathrm{~mL}(69.5$ \pm 11.3 vs $120.4 \pm 11.1, P<0.05$ ) (Fig. $1 \mathrm{~A})$. In contrast, the use of a probiotic alone (L. helveticus or B. longum) did not have any effect on the chronic stress induced-hypersensitivity at the volume of $0.08 \mathrm{~mL}$ and $0.10 \mathrm{~mL}$ of $\mathrm{CRD}$ (for example at $0.08 \mathrm{~mL}$ of $\mathrm{CRD}$, 
A
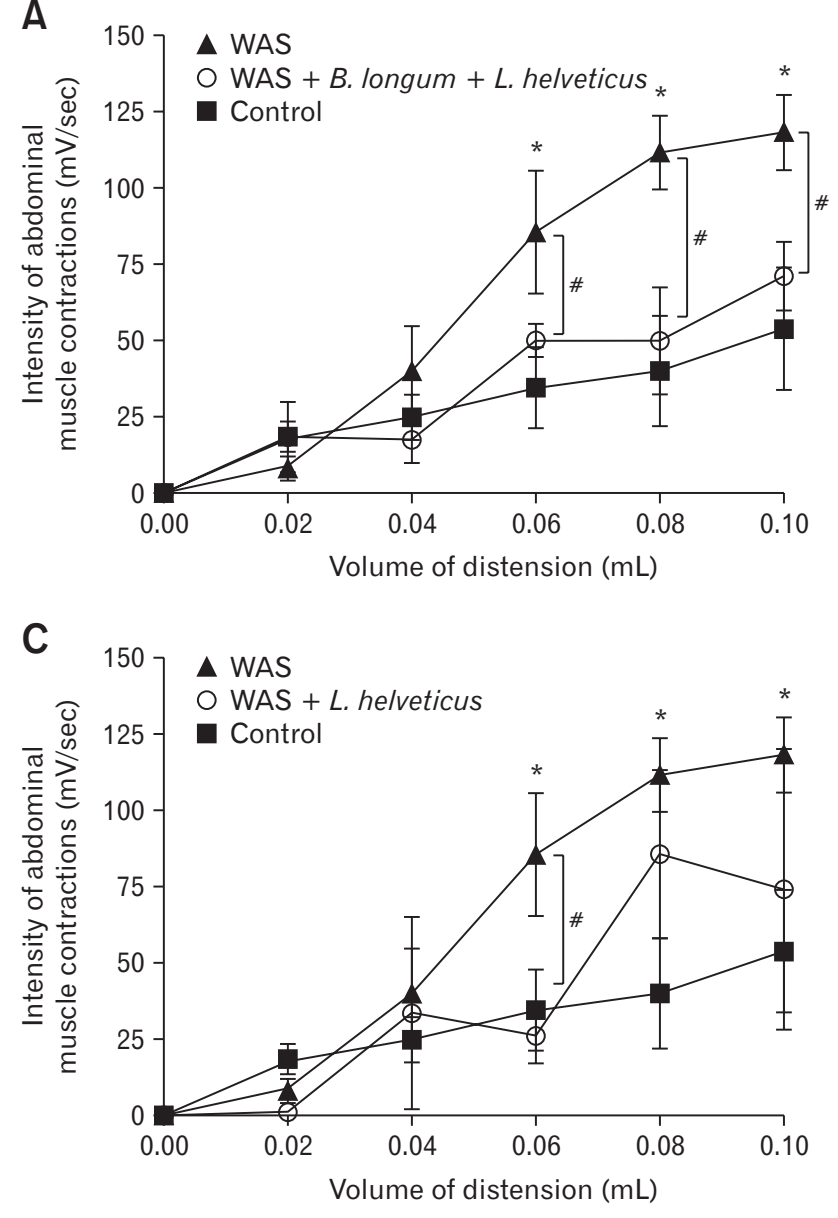

L. helveticus effect: $85.5 \pm 20.1$ vs $118.6 \pm 12.3$ or $B$. longum effect: $67.4 \pm 19.9$ vs $118.6 \pm 12.3)$. However, pretreatment with a single probiotic prevented the increase of abdominal pain induced by chronic stress at $0.06 \mathrm{~mL} \mathrm{CRD} \mathrm{volume} \mathrm{(B.} \mathrm{longum} \mathrm{and} L$. helveticus effect: respectively $37.7 \pm 9.1$ and $26.0 \pm 9.0$ vs $87.5 \pm$ 14.3) (Fig. 1B and 1C).

\section{Effect of the Probiotic Treatment on Stress-induced Increase of Neuroendocrine Hormone Levels}

In mice, WAS induced a significant increase of the plasma levels of corticosterone and catecholamines. There was no effect on stress hormone levels in unstressed mice (control group), whatever probiotic treatment was used. The probiotic combination (B. longum and L. helveticus) and B. longum treatment alone prevented the increase of these plasmatic stress markers. However, the $L$. helveticus treatment had no effect on the increase of either corticosterone or catecholamine concentrations in stressed mice (Table and Fig. 2).

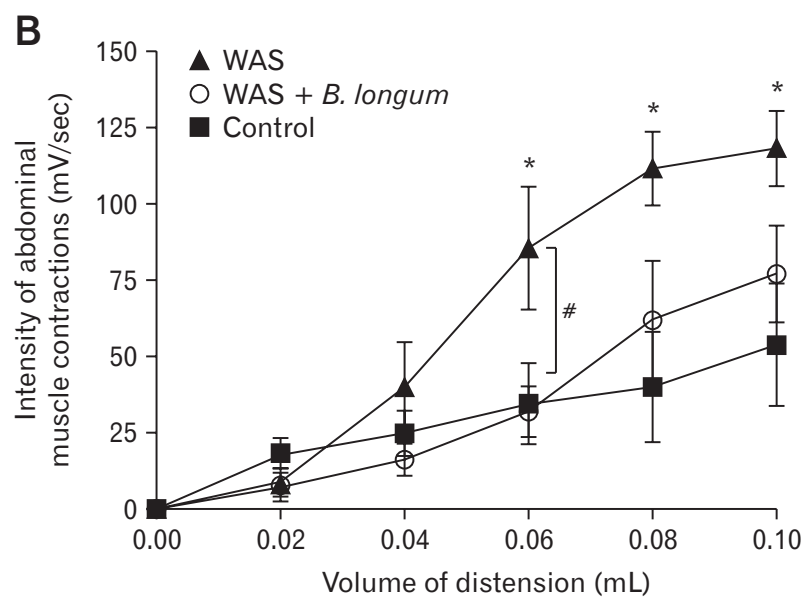

Figure 1. Prevention of abdominal pain induced by chronic stress (water avoidance stress [WAS]) depends on the strain probiotic. ${ }^{*} P$ $<0.05$ and ${ }^{\#} P<0.05$ are significantly different from control and WAS groups. B. longum, Bifidobacterium longum; L. helveticus, Lactobacillus helveticus.

Table. Effect of Probiotic Treatment or Vehicle on Plasma Levels of Noradrenaline and Adrenaline in Water Avoidance Stress (Chronic Stress) or Control Groups

\begin{tabular}{lcc}
\hline & \multicolumn{2}{c}{$\begin{array}{c}\text { Catecholamine concentration } \\
(\mathrm{ng} / \mathrm{mL})\end{array}$} \\
\cline { 2 - 3 } & \multicolumn{2}{c}{$\begin{array}{c}\text { Nonadrenaline } \\
\text { Adrenaline }\end{array}$} \\
\hline Control & $20 \pm 2$ & $19 \pm 2$ \\
Vehicle & $34 \pm 3$ & $13 \pm 2$ \\
B. longum + L. helveticus & $50 \pm 6$ & $20 \pm 3$ \\
B. longum & $14 \pm 2$ & $14 \pm 1$ \\
L. helveticus & & \\
WAS & $71 \pm 6^{\mathrm{a}}$ & $33 \pm 2^{\mathrm{a}}$ \\
Vehicle & $46 \pm 2^{\mathrm{b}}$ & $24 \pm 1^{\mathrm{b}}$ \\
B. longum + L. helveticus & $35 \pm 5^{\mathrm{b}}$ & $17 \pm 1^{\mathrm{b}}$ \\
B. longum & $52 \pm 5$ & $34 \pm 4$ \\
L. helveticus &
\end{tabular}

${ }^{a} P<0.05$ and ${ }^{b} P<0.05$ vs control + vehicle and WAS + vehicle group respectively.

WAS, water avoidance stress; $B$. longum, Bifidobacterium longum; $L$. helveticus, Lactobacillus helveticus.

Data are expressed as mean \pm SEM. 


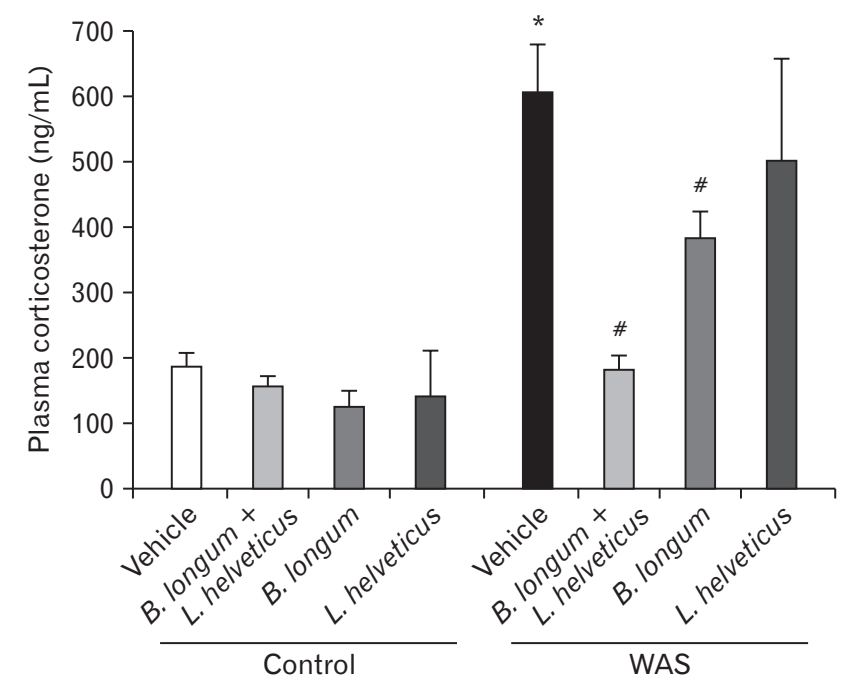

Figure 2. Probiotic treatments differentially prevent a plasma level of stress hormone markers. ${ }^{*} P<0.05$ and ${ }^{\#} P<0.05$ are significantly different from control and water avoidance stress (WAS; chronic stress). B. longum, Bifidobacterium longum; L. helveticus, Lactobacillus helveticus.

A

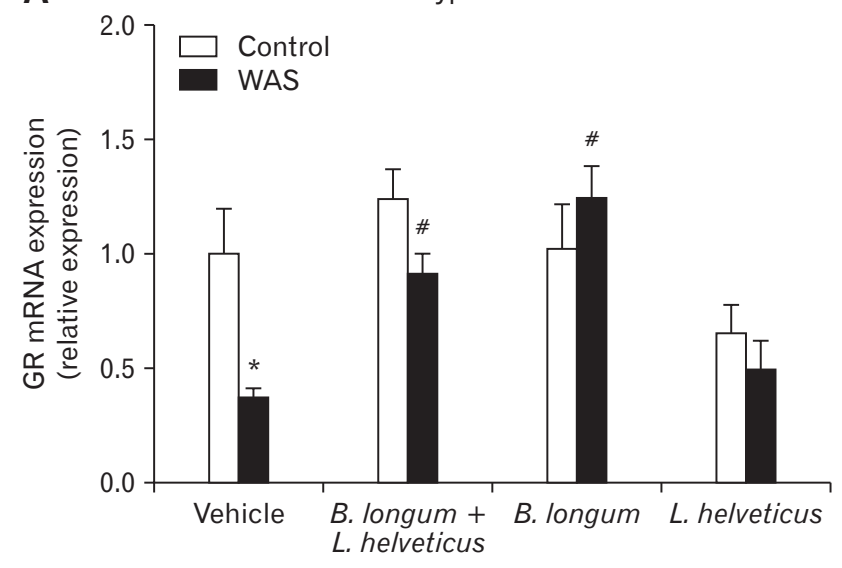

C

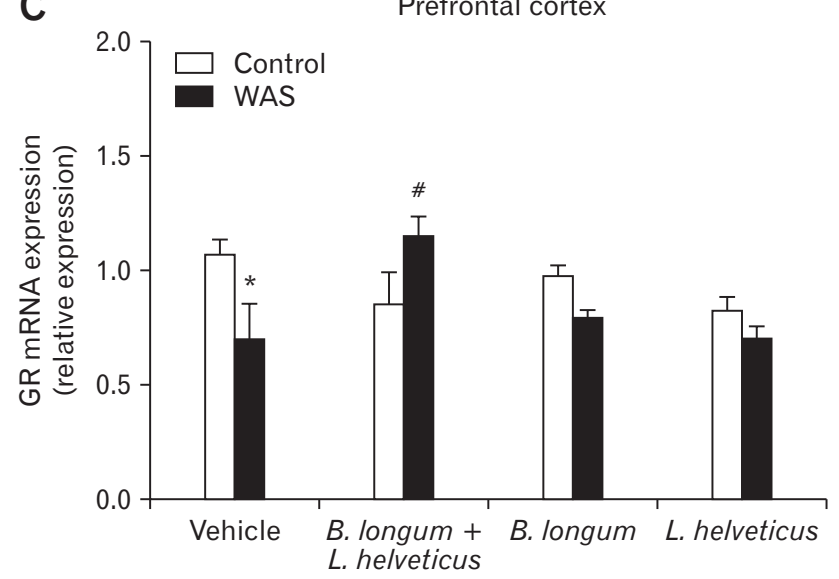

Differential Effects of Probiotic Treatment on Glucocorticoid Receptor Gene Expression in Stress-involved Brain Areas

The statistical analysis of the GR gene expression revealed a significantly lower expression in the WAS group compared to the control group. The difference was observed in all three brain areas: hypothalamus, hippocampus, and prefrontal cortex. The pre-treatment with the combination of 2 probiotics (B. longum and L. helveticus) or probiotic $B$. longum alone prevented the WAS-induced decrease of the GR gene expression in the hypothalamus (Fig. 3A) and hippocampus (Fig. 3B). The combination of these 2 probiotics blocked the stress-induced decrease of the GR gene expression in the prefrontal cortex, while probiotic B. longum did not (Fig. 3C). There was no significant effect on GR gene expression after pretreatment with $L$. helveticus alone in stressed mice.

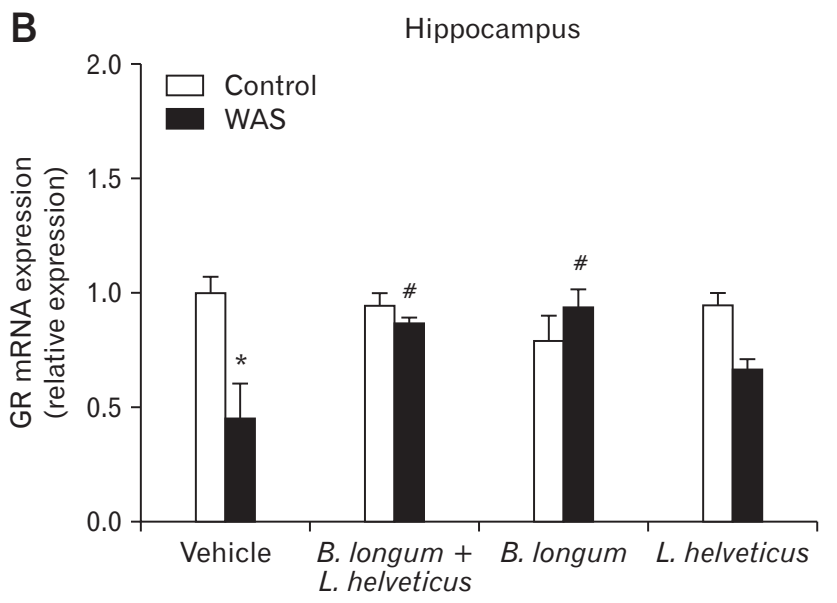

Figure 3. Specific probiotic treatment blocks chronic stress-induced decrease of glucocorticoid receptor (GR) gene expression in (A) hypothalamus, (B) hippocampus, and (C) prefrontal cortex. ${ }^{*} P<0.05$ and ${ }^{\#} P<0.05$ are significantly different from control and water avoidance stress (WAS; chronic stress). B. longum, Bifidobacterium longum; L. helveticus, Lactobacillus helveticus. 


\section{Discussion}

In the present study, we have shown that a 2 -week treatment with a combination of the 2 probiotics $B$. longum R0175 and $L$. helveticus R0052 decreases the chronic stress-induced visceral hypersensitivity in response to $\mathrm{CRD}$. Even if the probiotics $B$. longum or $L$. helveticus alone decrease the visceral pain induced by chronic stress only at low volume of $\mathrm{CRD}$, the data suggest that their association is more efficient. Furthermore, this study has shown that the combination of the 2 probiotics (B. longum and $L$. helveticus) or B.longum alone prevents the chronic stress-induced increase of plasma levels of stress hormones, whereas L. helveticus alone has no effect on these parameters. Interestingly, we have identified a decrease of GR gene expression in the hypothalamus and hippocampus after chronic stress, whereas the probiotic pre-treatment with the combination of the both probiotics ( $B$. longum and $L$. helveticus) and $B$. longum alone normalised GR gene expression. These findings are supported by our previous study showing that the combination of the 2 probiotics attenuates the stress response by modulating neuronal plasticity in specific brain areas. ${ }^{25}$ The effect of B.longum or L. helveticus probiotic treatment on the adverse stressful events, particularly on the visceral pain induced by chronic stress, depends on the glucocorticoid negative feedback regulation of the HPA axis. Finally, the combination of 2 probiotics ( $B$. longum and $L$. helveticus) has a better antinociceptive effect than the probiotic alone (B. longum or L. helveticus). This effect is probably due to the prevention of the increase of the stress hormone associated with the GR expression normalized and consequently, the regulation of negative feedback of hormone released in HPA axis.

Evidence studies in preclinical and human research suggest that the bidirectional signals sent between the CNS and the gastrointestinal tract influence stress reactivity. The gut microbiota seems to be a critical mediator of information in this brain-gut dialogue. $^{30-32}$ Indeed, the microbiome may influence neuronal circuitry involved in stress response, neuronal development and plasticity, pain perception, neurochemistry, or blood brain barrier integrity. ${ }^{33-35}$ Moreover, when gut microbiota in newborn rats was disrupted after antibiotic treatment, a visceral hypersensitivity was observed in adulthood and subsequently increased visceral pain signalling mechanism from the gastrointestinal tract. ${ }^{36}$ Clinical data showed that IBS patients had altered microbial profiles. ${ }^{11,12}$ Fecal microbiota transplanted from these patients into germ-free rats led to visceral hypersensitivity. ${ }^{15}$ Therefore, restoring the balance of gut microbiota by using the probiotics combination could be an interesting alterna- tive in the management of IBS symptoms. The physiological benefits of probiotics observed in IBS have highlighted the importance of using a cocktail of bacteria including the most effective probiotic strains. Some studies have shown that probiotic combination of lactobacillus and bifidobacterium species significantly decreases abdominal pain, ${ }^{37}$ delays the colonic transit, and reduces flatulence. ${ }^{38,39}$ In the current study, pre-treatment with $B$. longum or $L$. helveticus strains alone decreases visceral hypersensitivity induced by chronic stress in response to low noxious stimuli, but the combination of these 2 probiotics was more effective in stressed mice at all volumes of CRD. We have observed that the anti-nociceptive effect of probiotics depends on the probiotic strain used. The results have also shown a differential effect with respect to the influence of these different probiotic treatments on releasing plasma levels of stress hormones. Indeed, B. longum strain alone or in combination with L. helveticus strains decreased hypersecretion of corticosterone and catecholamine hormones under stressful conditions. However, the treatment with $L$. helveticus strain alone does not attenuate stress response in mice. The role of $L$. helveticus in the combination of probiotics can be explained by its anti-inflammatory properties. A recent study has shown that the $L$. helveticus strain effect depends on gut inflammatory state and elicits anti-inflammatory responses in intestinal inflammation-associated behaviour. ${ }^{40}$ The anti-inflammatory properties of $L$. helveticus are probably due to its capacity to decrease pathogen adhesion to the epithelial cells. ${ }^{41}$ On the other hand, $B$. longum probiotic strain enhances some positive effects on the cognition by decreasing anxiety in mice. ${ }^{42,43}$ Taking into account the specificity of action of each strain at the peripheral and central level, all these data converge to show that CNS function is positively impacted by the combination of these 2 probiotics (B. longum and $L$. helveticus).

An exacerbated HPA axis in IBS patients after hormone challenge correlated with visceral pain and a reduction in GR protein expression in peripheral blood mononuclear cells. ${ }^{44}$ Although, it has been shown in adult rats that an early life stress evokes a change of GR expression in the hippocampus and central amygdala, ${ }^{45,46}$ which enhances an activation of somatosensory area and visceral hypersensitivity. ${ }^{47}$ Moreover, in postnatal day 10, a single stress session for 4 hours induced an alteration of GR expression in the colon in pups, weakened the intestinal barrier integrity allowing bacterial translocation, ${ }^{48}$ and subsequently provoked later visceral hypersensitivity. ${ }^{49}$ The present study reveals that the treatment with the combination of 2 probiotics $L$. helveticus and B. longum or $B$. longum alone restores the chronic stress-induced decrease in hippocampal and hypothalamic GR expression. In addition to the 
previous observations on the plasma level of stress hormones, the effect of probiotics on GR expression in several brain areas seems to participate in the regulation of visceral hypersensitivity. We hypothesize that the specific probiotic treatment counteracts the disrupted glucocorticoid signalling, impaired negative feedback, and pain. In fact, endogenous hormonal interaction with bacterial products released by probiotic and/or bacteria in the intestinal microenvironment can be suggested as a mechanism of action of the probiotic on the alleviation of visceral pain. Data from germ free rodents suggest that hormonal status plays an important role in the host-microbial interaction. Host hormonal signals are able to shape the behaviour of the gut microbiome, and in return, the microbiome residents signal the changes to their host and can affect physical and even mental well-being. ${ }^{50}$ For example, endogenous hormones such as catecholamines enhance bacterial adhesion of pathogens to the host tissues $^{51}$ or downregulate resistance of pathogens of host microbial peptides. $^{52}$ Another study has shown that the human sex hormones decrease bacterial virulence by inhibiting quorum sensing, which is a specific communication between the bacterial and neuroendocrine systems. ${ }^{53}$ Thereby, host neuroendocrine hormones sensing modifies indirectly or directly the gut environment. Thus, the observed effects of the probiotic strains on visceral hypersensitivity induced by chronic stress prove the importance of selecting a combination of "good" bacteria for endocrine-microbiome crosstalk. These findings highlight one of the mechanisms by which microbiome residents under probiotic influence can sense the emotional status of their host.

This study shows that the effect of probiotics on stress-induced hypersensitivity is strain specific. The treatment with the combination of the 2 probiotic strains (B. longum and L. helveticus) prevents the perception of visceral pain associated with an attenuation of stress hormones, and has a potential effect on GR expression. However, the probiotic treatment with only $L$. helveticus or $B$. longum is not effective to reduce chronic stress-induced hypersensitivity in response to all volumes of CRD. This differential effect correlated with the attenuation of stress hormone associated with a change of GR expression in specific areas involved in stress response.

We speculate that the combination of these probiotics may regulate the negative feedback of the HPA axis by reducing glucocorticoid signaling in the limbic system, particularly in the hippocampus. Finally, the prevention of increased stress hormone levels plays an important role in the attenuation of visceral pain induced by chronic stress. This study supports strongly the probiotic formulation Probio'Stick (B. longum R0175 and L. helveticus R0052) as a therapeutic solution for treating chronic stress and associated visceral pain.

\section{Financial support: None.}

\section{Conflicts of interest: None.}

Author contributions: Conceived and designed the experiments: Isabelle Payard, Claire Rolland, Cherryl Harkat, and Afifa Ait-Belgnaoui; performed the experiments: I Isabelle Payard, Claire Rolland, and Cherryl Harkat; analysed and interpretation: Viorica Braniste and Afifa Ait-Belgnaoui; wrote the manuscript: Afifa AitBelgnaoui; and critical revision of manuscript: Viorica Braniste, Vassillia Théodorou, and Thomas Tompkins.

\section{References}

1. Canavan C, West J, Card T. The epidemiology of irritable bowel syndrome. Clinical Epidemiol 2014;6:71-80.

2. Chey WD, Kurlander J, Eswaran S. Irritable bowel syndrome: a clinical review. JAMA 2015;313:949-958.

3. Whitehead WE, Crowell MD, Robinson JC, Heller BR, Schuster MM. Effects of stressful life events on bowel symptoms: subjects with irritable bowel syndrome compared with subjects without bowel dysfunction. Gut 1992;33:825-830.

4. Moloney RD, O'Mahony SM, Dinan TG, Cryan JF. Stress-induced visceral pain: toward animal models of irritable-bowel syndrome and associated comorbidities. Frontiers Psychiatry 2015;6:15.

5. Thijssen AY, Jonkers DM, Leue C, et al. Dysfunctional cognitions, anxiety and depression in irritable bowel syndrome. J Clin Gastroenterol 2010;44:e236-e241.

6. Pacák K, Palkovits M. Stressor specificity of central neuroendocrine responses: implications for stress-related disorders. Endocr Rev 2001;22:502-548.

7. Watts AG. Glucocorticoid regulation of peptide genes in neuroendocrine CRH neurons: a complexity beyond negative feedback. Front Neuroendocrinol 2005;26:109-130.

8. Harris AP, Holmes MC, de Kloet ER, Chapman KE, Seckl JR. Mineralocorticoid and glucocorticoid receptor balance in control of HPA axis and behaviour. Psychoneuroendocrinology 2013;38:648-658.

9. Mizoguchi K, Ishige A, Aburada M, Tabira T. Chronic stress attenuates glucocorticoid negative feedback: involvement of the prefrontal cortex and hippocampus. Neuroscience 2003;119:887-897.

10. Johnson AC, Tran L, Schulkin J, Greenwood-Van Meerveld B. Importance of stress receptor-mediated mechanisms in the amygdala on visceral pain perception in an intrinsically anxious rat. Neurogastroenterol Motil 2012;24:479-486, e219.

11. Dinan TG, Cryan JF. Regulation of the stress response by the gut microbiota: implications for psychoneuroendocrinology. Psychoneuroendocrinology 2012;37:1369-1378. 
12. Jeffery IB, O’Toole PW, Öhman L, et al. An irritable bowel syndrome subtype defined by species-specific alterations in faecal microbiota. Gut 2012;61:997-1006.

13. Rajilić-Stojanović M, Biagi E, Heilig HG, et al. Global and deep molecular analysis of microbiota signatures in fecal samples from patients with irritable bowel syndrome. Gastroenterology 2011;141:1792-1801.

14. Tap J, Derrien M, Törnblom H, et al. Identification of an intestinal microbiota signature associated with severity of irritable bowel syndrome. Gastroenterology 2017;152:111-123, e8.

15. Crouzet L, Gaultier E, Del'Homme C, et al. The hypersensitivity to colonic distension of IBS patients can be transferred to rats through their fecal microbiota. Neurogastroenterol Motil 2013;25:e272-e282.

16. Vrieze A, Van Nood E, Holleman F, et al. Transfer of intestinal microbiota from lean donors increases insulin sensitivity in individuals with metabolic syndrome. Gastroenterology 2012;143:913-916, e7.

17. Suskind DL, Brittnacher MJ, Wahbeh G, et al. Fecal microbial transplant effect on clinical outcomes and fecal microbiome in active Crohn's disease. Inflamm Bowel Dis 2015;21:556-563.

18. Pinn DM, Aroniadis OC, Brandt LJ. Is fecal microbiota transplantation the answer for irritable bowel syndrome? A single-center experience. Am J Gastroenterol 2014;109:1831-1832.

19. Mazurak N, Broelz E, Storr M, Enck P. Probiotic therapy of the irritable bowel syndrome: why is the evidence still poor and what can be done about it? J Neurogastroenterol Motil 2015;21:471-485.

20. Sisson G, Ayis S, Sherwood RA, Bjarnason I. Randomised clinical trial: a liquid multi-strain probiotic vs. placebo in the irritable bowel syndrome--a 12 week double-blind study. Aliment Pharmacol Ther 2014;40:51-62.

21. Kamiya T, Wang L, Forsythe P, et al. Inhibitory effects of Lactobacillus reuteri on visceral pain induced by colorectal distension in SpragueDawley rats. Gut 2006;5 5:191-196.

22. Ma X, Mao YK, Wang B, Huizinga JD, Bienenstock J, Kunze W. Lactobacillus reuteri ingestion prevents hyperexcitability of colonic DRG neurons induced by noxious stimuli. Am J Physiol Gastrointest Liver 2009;296:G868-G875.

23. McKernan DP, Fitzgerald P, Dinan TG, Cryan JF. The probiotic Bifidobacterium infantis 35624 displays visceral antinociceptive effects in the rat. Neurogastroenterol Motil 2010;22:1029-1035, e268.

24. Gareau MG, Jury J, MacQueen G, Sherman PM, Perdue MH. Probiotic treatment of rat pups normalises corticosterone release and ameliorates colonic dysfunction induced by maternal separation. Gut 2007;56:1522-1528.

25. Ait-Belgnaoui A, Colom A, Braniste V, et al. Probiotic gut effect prevents the chronic psychological stress-induced brain activity abnormality in mice. Neurogastroenterol Motil 2014;26:510-520.

26. Zheng G, Wu SP, Hu Y, Smith DE, Wiley JW, Hong S. Corticosterone mediates stress-related increased intestinal permeability in a regionspecific manner. Neurogastroenterol Motil 2013;25:e127-e139.

27. Zheng G, Hong S, Hayes JM, Wiley JW. Chronic stress and peripheral pain: evidence for distinct, region-specific changes in visceral and somatosensory pain regulatory pathways. Exp Neurol 2015;273:301-311.

28. Fourie NH, Wang D, Abey SK, et al. Structural and functional alterations in the colonic microbiome of the rat in a model of stress induced irritable bowel syndrome. Gut microbes 2017;8:33-45.

29. Annaházi A, Dabek M, Gecse K, et al. Proteinase-activated receptor-4 evoked colorectal analgesia in mice: an endogenously activated feedback loop in visceral inflammatory pain. Neurogastroenterol Motil 2012;24:76-85, e13.

30. Moloney RD, Desbonnet L, Clarke G, Dinan TG, Cryan JF. The microbiome: stress, health and disease. Mamm Genome 2014;25:49-74.

31. Grenham S, Clarke G, Cryan JF, Dinan TG. Brain-gut-microbe communication in health and disease. Front Physiol 2011;2:94.

32. Collins SM, Surette M, Bercik P. The interplay between the intestinal microbiota and the brain. Nat Rev Microbiol 2012;10:735-742.

33. Borre YE, Moloney RD, Clarke G, Dinan TG, Cryan JF. The impact of microbiota on brain and behavior: mechanisms \& therapeutic potential. Adv Exp Med Biol 2014;817:373-403.

34. Luczynski P, McVey Neufeld KA, Oriach CS, Clarke G, Dinan TG, Cryan JF. Growing up in a bubble: using germ-free animals to assess the influence of the gut microbiota on brain and behavior. Int J Neuropsychopharmacol 2016;19:pyw020.

35. Braniste V, Al-Asmakh M, Kowal C, et al. The gut microbiota influences blood-brain barrier permeability in mice. Science Transl Med 2014;6:263ra158.

36. O'Mahony SM, Felice VD, Nally K, et al. Disturbance of the gut microbiota in early-life selectively affects visceral pain in adulthood without impacting cognitive or anxiety-related behaviors in male rats. Neuroscience 2014;277:885-901.

37. Saggioro A. Probiotics in the treatment of irritable bowel syndrome. J Clin Gastroenterol 2004;38(6 suppl):S104-S106.

38. Kim HJ, Camilleri M, McKinzie S, et al. A randomized controlled trial of a probiotic, VSL\#3, on gut transit and symptoms in diarrhoeapredominant irritable bowel syndrome. Aliment Pharmacol Ther 2003;17:895-904.

39. Kim HJ, Vazquez Roque MI, Camilleri M, et al. A randomized controlled trial of a probiotic combination VSL\# 3 and placebo in irritable bowel syndrome with bloating. Neurogastroenterol Motil 2005;17:687696.

40. Ohland CL, Kish L, Bell H, et al. Effects of Lactobacillus helveticus on murine behavior are dependent on diet and genotype and correlate with alterations in the gut microbiome. Psychoneuroendocrinology 2013;38:1738-1747.

41. Johnson-Henry KC, Hagen KE, Gordonpour M, Tompkins TA, Sherman PM. Surface-layer protein extracts from Lactobacillus helveticus inhibit enterohaemorrhagic Escherichia coli O157:H7 adhesion to epithelial cells. Cell Microbiol 2007;9:356-367.

42. Savignac HM, Tramullas M, Kiely B, Dinan TG, Cryan JF. Bifidobacteria modulate cognitive processes in an anxious mouse strain. Behav Brain Res 2015;287:59-72.

43. Savignac HM, Kiely B, Dinan TG, Cryan JF. Bifidobacteria exert strainspecific effects on stress-related behavior and physiology in BALB/c mice. Neurogastroenterol Motil 2014;26:1615-1627.

44. Videlock EJ, Shih W, Adeyemo M, et al. The effect of sex and irritable bowel syndrome on HPA axis response and peripheral glucocorticoid receptor expression. Psychoneuroendocrinology 2016;69:67-76. 
45. Zhang G, Zhao BX, Hua R, et al. Hippocampal microglial activation and glucocorticoid receptor down-regulation precipitate visceral hypersensitivity induced by colorectal distension in rats. Neuropharmacology 2016;102:295-303.

46. Prusator DK, Greenwood-Van Meerveld B. Amygdala-mediated mechanisms regulate visceral hypersensitivity in adult females following early life stress: importance of the glucocorticoid receptor and corticotropinreleasing factor. Pain 2017;158:296-305.

47. Wouters MM, Van Wanrooy S, Casteels C, et al. Altered brain activation to colorectal distention in visceral hypersensitive maternal-separated rats. Neurogastroenterol Motil 2012;24:678-685, e297.

48. Moussaoui N, Braniste V, Ait-Belgnaoui A, et al. Changes in intestinal glucocorticoid sensitivity in early life shape the risk of epithelial barrier defect in maternal-deprived rats. PLoS One 2014;9:e88382.

49. Barreau F, Cartier C, Ferrier L, Fioramonti J, Bueno L. Nerve growth factor mediates alterations of colonic sensitivity and mucosal barrier induced by neonatal stress in rats. Gastroenterology 2004;127:524-534.

50. Sandrini S, Aldriwesh M, Alruways M, Freestone P. Microbial endocrinology: host-bacteria communication within the gut microbiome. J Endocrinol 2015;225:R21-R34.

51. Hegde M, Wood TK, Jayaraman A. The neuroendocrine hormone norepinephrine increases Pseudomonas aeruginosa PA14 virulence through the las quorum-sensing pathway. Applied microbiology and biotechnology 2009;84:763-776.

52. Karavolos MH, Winzer K, Williams P, Khan CM. Pathogen espionage: multiple bacterial adrenergic sensors eavesdrop on host communication systems. Mol Microbiol 2013;87:455-465.

53. Beury-Cirou A, Tannières M, Minard C, et al. At a supra-physiological concentration, human sexual hormones act as quorum-sensing inhibitors. PLoS One 2013;8:e83564. 